\title{
ARTICLE Sevoflurane postconditioning protects against myocardial ischemia/reperfusion injury by restoring autophagic flux via an NO-dependent mechanism
}

\author{
Shi-gang Qiao ${ }^{1,2,3}$, Ying Sun ${ }^{4}$, Bo Sun ${ }^{4}$, An Wang ${ }^{1,4}$, Jia Qiu ${ }^{1,4}$, Lei Hong ${ }^{2}$, Jian-zhong $\mathrm{An}^{2}$, Chen Wang ${ }^{2,3}$ and Hui-ling Zhang ${ }^{1}$
}

\begin{abstract}
Volatile anesthetics improve postischemic cardiac function and reduce infarction even when administered for only a brief time at the onset of reperfusion. A recent study showed that sevoflurane postconditioning (SPC) attenuated myocardial reperfusion injury, but the underlying mechanisms remain unclear. In this study, we examined the effects of sevoflurane on nitric oxide (NO) release and autophagic flux during the myocardial ischemia/reperfusion (I/R) injury in rats in vivo and ex vivo. Male rats were subjected to $30 \mathrm{~min}$ ischemia and $2 \mathrm{~h}$ reperfusion in the presence or absence of sevoflurane (1.0 minimum alveolar concentration) during the first $15 \mathrm{~min}$ of reperfusion. We found that SPC significantly improved hemodynamic performance after reperfusion, alleviated postischemic myocardial infarction, reduced nicotinamide adenine dinucleotide content loss, and cytochrome $c$ release in heart tissues. Furthermore, SPC significantly increased the phosphorylation of endothelial nitric oxide synthase (NOS) and neuronal nitric oxide synthase, and elevated myocardial NOS activity and NO production. All these effects were abolished by treatment with an NOS inhibitor NG-nitro-L-arginine methyl ester (L-NAME, $10 \mathrm{mg} / \mathrm{kg}$, i.v.). We also observed myocardial I/R-induced accumulation of autophagosomes in heart tissues, as evidenced by increased ratios of microtubule-associated protein 1 light chain 3 II/l, upregulation of Beclin 1 and P62, and reduced lysosome-associated membrane protein-2 expression. SPC significantly attenuated I/Rimpaired autophagic flux, which were blocked by L-NAME. Moreover, pretreatment with the autophagic flux blocker chloroquine $(10 \mathrm{mg} / \mathrm{kg}$, i.p.) increased autophagosome accumulation in SPC-treated heart following I/R and blocked SPC-induced cardioprotection. The same results were also observed in a rat model of myocardial I/R injury ex vivo, suggesting that SPC protects rat hearts against myocardial reperfusion injury by restoring I/R-impaired autophagic flux via an NO-dependent mechanism.
\end{abstract}

Keywords: myocardial reperfusion injury; autophagy; nitric oxide; nitric oxide synthase; sevoflurane; NG-nitro-L-arginine methyl ester; chloroquine

Acta Pharmacologica Sinica (2019) 40:35-45; https://doi.org/10.1038/s41401-018-0066-y

\section{INTRODUCTION}

Coronary artery ischemic disease remains a major cause of death and morbidity worldwide. Restoration of the blood supply to the ischemic myocardium is essential for limiting the damage caused by acute myocardial infarction. In clinical practice, cardiac surgery causes myocardial ischemia, resulting in cardiac damage. In the reperfusion phase, the myocardium is injured again upon restoration of blood flow. Because reperfusion induces damage, this process is referred to as reperfusion injury $[1,2]$. Volatile anesthetics improve postischemic cardiac function and reduce infarction even when administered for only a brief time at the onset of reperfusion. This phenomenon is similar to ischemic postconditioning and is called anesthetic postconditioning [3]. Recently, a study found that a combined isoflurane preconditioning and propofol postconditioning regimen synergistically attenuated myocardial reperfusion injury in patients [4]. Although basic and clinical studies have shown that anesthetic postconditioning with agents such as sevoflurane is an effective strategy to reduce myocardial injury, the precise mechanisms have not been completely elucidated.

Autophagy is generally considered to be involved in both classic ischemic postconditioning- and pharmacologic postconditioning-mediated cardioprotection [5, 6]. Ischemic postconditioning has been reported to decrease oxidative stress and myocardial ischemia/reperfusion (I/R) injury partially by enhanced autophagy clearance and autophagy activity during early reperfusion. Activation of the neuronal nitric oxide synthase (nNOS)/ AMPK/mTOR pathway is involved in this cardioprotective effect [7]. By reusing degraded cellular proteins and organelles to maintain mitochondrial ATP production and homeostasis, autophagy may promote cell survival. However, excessive activation of autophagic pathways may also result in cell death and exacerbate damage following I/R [8]. Maintenance of mitochondrial function

\footnotetext{
'Department of Pharmacology and Laboratory of Cerebrovascular and Cardiovascular Pharmacology, College of Pharmaceutical Science, Soochow University, Suzhou 215123, China; ${ }^{2}$ Institute of Clinical Medicine Research, the Affiliated Suzhou Hospital of Nanjing Medical University; Suzhou Science and Technology Town Hospital, Suzhou 215153,

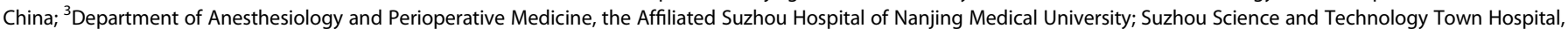
Suzhou 215153, China and ${ }^{4}$ Department of Anesthesiology, the Second Affiliated Hospital of Soochow University, Suzhou 215004, China Correspondence: Chen Wang (anesthesia_chen@163.com) or Hui-ling Zhang (huilingzhang07@hotmail.com)
} 
is important to inhibit excessive autophagosome induction and accumulation as mitochondrial dysfunction is associated with dramatic activation of autophagy during myocardial I/R [9]. Moreover, autophagic flux is a dynamic process that starts with autophagosome formation induction and ends with autophagosome degradation in lysosomes [10]. In cardiac I/R injury, mitochondrial dysfunction induces autophagosome accumulation, thus contributing to cardiomyocyte death [11]. Although previous reports have shown that sevoflurane postconditioning (SPC) restored autophagic flux in the myocardium and thus provided cardioprotection $[12,13]$, the precise mechanism requires further elucidation.

In addition, anesthetics have been considered to mimic ischemic preconditioning and ischemic postconditioning by increasing nitric oxide (NO), thus improving mitochondrial function, maintaining the ATP content, and reducing the expression of cytochrome $c[14,15]$. NO is a signaling molecule that serves as an upstream effector in mitochondria-mediated cell death pathways during myocardial $\mathrm{I} / \mathrm{R}$, including antagonism of beta-adrenergic stimulation, activation of $\mathrm{K}_{\text {ATP }}$ channels and cyclooxygenase-2, inhibition of calcium channels and proapoptotic proteins, and antioxidant effects [16]. Furthermore, a recent study showed that an endothelial nitric oxide synthase (eNOS)dependent mechanism involved in anesthetic postconditioning conferred cardioprotection by preventing mitochondrial permeability transition pore opening [17]. NO-induced autophagy protects human dental pulp cells and hepatic stellate cells against apoptosis $[18,19]$. These studies collectively suggest that NO may play a fundamental biological role in anesthetic postconditioning by mediating restoration of autophagic flux. In this study, we explored whether increased NO release due to sevoflurane exposure at the onset of reperfusion is involved in the restoration of autophagic flux in myocardial I/R injury and plays a key role in SPC-induced cardioprotection against reperfusion injury.

\section{MATERIALS AND METHODS}

Animals

Adult male Sprague-Dawley rats (weight: $300 \pm 50 \mathrm{~g}$; age: 9-12 weeks) were purchased from the Animal Center of Soochow University (Suzhou, China). All animals were maintained under a $12 \mathrm{~h}$ light-dark cycle in a temperature-controlled room and were allowed free access to water and food for 1 week prior to euthanasia. The experimental procedures were approved by the Animal Care and Use Committee of the Medical College of Soochow University and conformed to the Guide for the Care and Use of Laboratory Animals (protocol number: SZULL-20090309, approved on March 9, 2009).

\section{Myocardial I/R injury ex vivo}

The rats were anesthetized with sodium pentobarbital $(50 \mathrm{mg} / \mathrm{kg})$ and heparinized (heparin, $1000 \mathrm{U} / \mathrm{kg}$ ) intraperitoneally. Their hearts were removed promptly, mounted on a Langendorff apparatus, and perfused retrogradely through the aorta at a constant pressure of $80 \mathrm{mmHg}$ with Krebs-Henseleit (K-H) buffer $\left(\mathrm{NaCl}, 118 \mathrm{mM} ; \mathrm{NaHCO}_{3}, 25 \mathrm{mM} ; \mathrm{KCl}, 4.8 \mathrm{mM} ; \mathrm{MgSO}_{4}, 1.2 \mathrm{mM}\right.$; $\mathrm{KH}_{2} \mathrm{PO}_{4}, 1.2 \mathrm{mM} ; \mathrm{CaCl}_{2}, 2.0 \mathrm{mM}$; Glucose: $11 \mathrm{mM}$ ) at $37^{\circ} \mathrm{C}$ as previously described $[2,19]$. A saline-filled latex balloon connected to a pressure transducer (YP200, Beijing SIA Industrial Electronies $\mathrm{CO}, \mathrm{Ltd})$ by a polyethylene tube was inserted into the left ventricle via the left auricle incision. The experiments were assigned to the following five groups ( $n=8 /$ group): Sham, I/R, SPC, NG-nitro-Larginine methyl ester (L-NAME) and SPC + L-NAME (Fig. 1a). The sham operation group (Sham) was continuously perfused for 180 min with $\mathrm{K}-\mathrm{H}$ buffer without $\mathrm{I} / \mathrm{R}$. All rat hearts were stabilized for $30 \mathrm{~min}$ and then subjected to $30 \mathrm{~min}$ of no-flow global ischemia, followed by $120 \mathrm{~min}$ of reperfusion with or without 1.0 minimum alveolar concentration of sevoflurane exposure (I/R and SPC groups, respectively) in the absence or presence $100 \mu \mathrm{M}$ of L-NAME (L-NAME and SPC + L-NAME groups, respectively). a

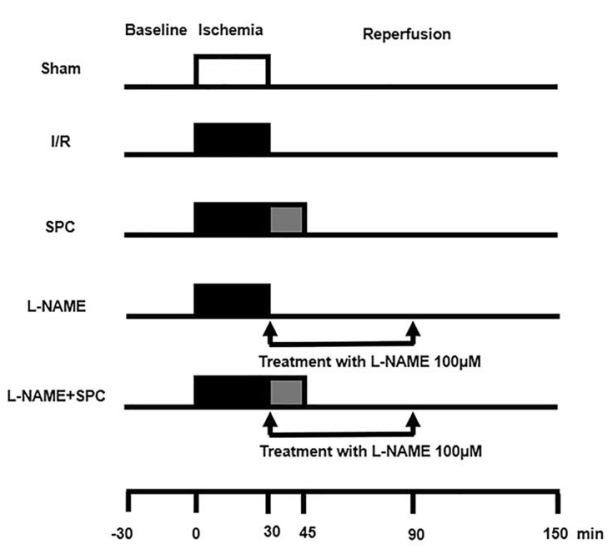

$\mathrm{b}$

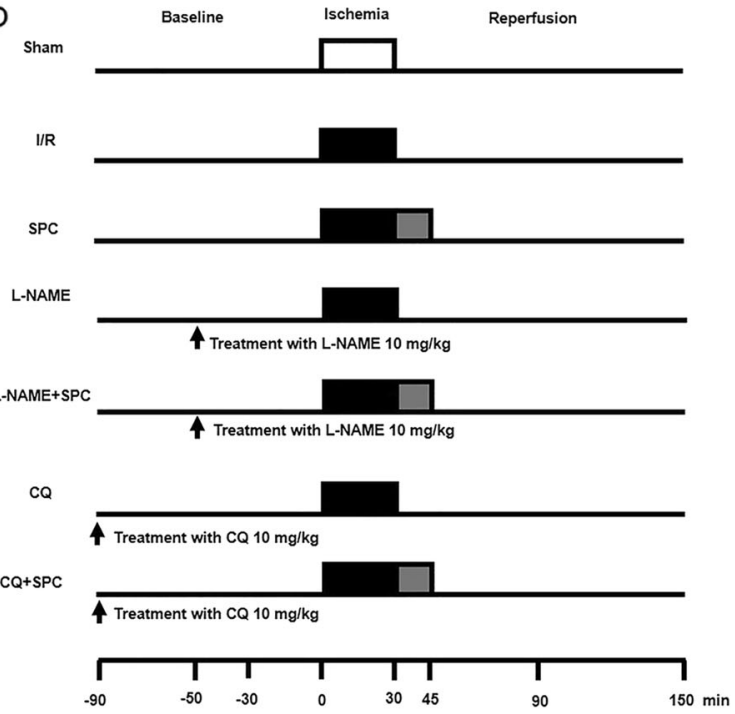

Ischemia

- Drug administration

Fig. 1 Schematic illustration of the experimental protocol for sevoflurane postconditioning (SPC). All rats underwent 30 min of ischemia followed by $120 \mathrm{~min}$ of reperfusion. The group underwent a time-matched procedure without ischemia. Sevoflurane at $2.5 \%$ was perfused ex vivo or inhaled in vivo at initial reperfusion for $15 \mathrm{~min}$. The putative nitric oxide synthase (NOS) inhibitor NG-nitro-L-arginine methyl ester (L-NAME) was continuously perfused for $60 \mathrm{~min}$ immediately following the ischemia in protocol (a) (ex vivo experiments), and L-NAME and chloroquine (CQ) were administered for 10 min starting from $50 \mathrm{~min}$ and 90 min before ischemia, respectively, in protocol (b) (in vivo experiments) 
L-NAME was applied at the onset of reperfusion for $60 \mathrm{~min}$. Sevoflurane was bubbled into K-H buffer using a Dräger vaporizer placed in the $95 \% \mathrm{O}_{2} / 5 \% \mathrm{CO}_{2}$ gas mixture line with a $\mathrm{pH}$ of 7.35-7.45 at the onset of reperfusion for $15 \mathrm{~min}$. Sevoflurane concentrations in the coronary effluent were determined by gas chromatography. Left ventricular developed pressure (LVDP), left ventricular end-diastolic pressure (LVEDP), the maximum rate of increase in $\operatorname{LVDP}(+\mathrm{d} P / \mathrm{d} t)$ and the maximum rate of decrease in LVDP $(-\mathrm{dP} / \mathrm{d} t)$ at baseline, $20 \mathrm{~min}$ after ischemia, and 10, 30, 60, 90, and $120 \mathrm{~min}$ after reperfusion were recorded. LVDP, LVEDP and $\pm \mathrm{d} P / \mathrm{d} t$ are major indices of myocardial contractility and relaxation, and LVEDP is not only a marker of diastolic function but also a good predictor of cardiac mortality $[2,15]$.

\section{Myocardial I/R injury in vivo}

Myocardial I/R injury models were produced by occluding the left anterior descending coronary artery (LAD) as previously described $[20,21]$. Briefly, rats were anesthetized with intraperitoneal sodium pentobarbital $(50 \mathrm{mg} / \mathrm{kg})$ and received additional sodium pentobarbital $(25 \mathrm{mg} / \mathrm{kg}$ ) every $60-90 \mathrm{~min}$ to ensure that pedal and palpebral reflexes were absent throughout the experiment. The right jugular vein was cannulated to infuse drugs and saline, and the right carotid artery was cannulated to measure hemodynamic variables. A tracheostomy was performed through a ventral midline incision, and the trachea was cannulated. The rats were mechanically ventilated at 55-60 breaths per minute with a 33\% air-oxygen mixture using a rodent ventilator (Shanghai Alcott Biotech, Shanghai, China). Hypercarbia or hypoxia was managed according to arterial blood gas analyses (Istat, Princeton, NJ, USA) and body temperature was maintained at $37 \pm 0.5^{\circ} \mathrm{C}$ using a heating pad. Myocardial ischemia was induced by occluding the LAD via suture, and reperfusion was initiated by loosening the suture. Successful myocardial ischemia was confirmed by a cyanosis response in the ventricular surface and marked arrhythmia. A heparin-filled polyethylene catheter was placed into the right carotid artery and connected to a pressure transducer coupled to a data acquisition system (Medlab-U/4C501H system, Nanjing Meiyi Technology, China) to measure hemodynamic data, including the heart rate, mean arterial blood pressure and rate pressure product, at $30 \mathrm{~min}$ of equilibration $\left(T_{0}\right)$ and at $30 \mathrm{~min}\left(T_{1}\right), 60 \mathrm{~min}\left(T_{2}\right), 90 \mathrm{~min}\left(T_{3}\right)$, and $2 \mathrm{~h}\left(T_{4}\right)$ after reperfusion.

The experimental design is illustrated in Fig. 1b. Baseline hemodynamics and arterial blood gas tensions were recorded 30 min after instrumentation was completed. The rats receiving $33 \%$ oxygen without subsequent I/R served as the Sham group. All other rats underwent LAD occlusion for $30 \mathrm{~min}$ followed by 120 min of reperfusion with or without 1.0 minimum alveolar concentration of sevoflurane exposure at the onset of reperfusion for $15 \mathrm{~min}$. The nonselective NO synthase (NOS) inhibitor (L-NAME, $10 \mathrm{mg} / \mathrm{kg}$ ) was administered via the right jugular vein, and the autophagic flux blocker chloroquine $(C Q, 10 \mathrm{mg} / \mathrm{kg}$ ) was administered via intraperitoneal injection. L-NAME and CQ were dissolved in $1 \mathrm{~mL}$ of $0.9 \%$ saline; L-NAME was injected slowly over $10 \mathrm{~min}$ 50 min before LAD occlusion, while CQ was administered $90 \mathrm{~min}$ before LAD occlusion.

Myocardial infarct size

Myocardial infarct size was determined by 2,3,5-triphenyltetrazolium chloride triazole (TTC) staining. The perfused hearts were removed rapidly and cut into six transverse slices of 2-mm thickness at the end of the $2 \mathrm{~h}$ reperfusion period, as shown in Fig. 2a, b. The coronary artery was immediately re-occluded, and Evans blue solution ( $5 \%$, Sigma-Aldrich, St. Louis, MO, USA) was administered via the right jugular vein to stain the nonischemic region of the left ventricle after $2 \mathrm{~h}$ of reperfusion, as shown in Fig. 1b. The slices were incubated in phosphate buffer (PBS, pH 7.4) containing $1 \%$ TTC (Sigma-Aldrich Co., St. Louis, MO, USA) at $37^{\circ} \mathrm{C}$ for $15 \mathrm{~min}$ and subsequently fixed overnight in $10 \%$ formaldehyde solution ( $\mathrm{pH} 7.4)$ for $24 \mathrm{~h}$. The infarct size was calculated as the percentage of the total range of the left ventricle area at risk (IS/AAR) using Alpha View gel image analysis software (Alpha Ease FC, USA).

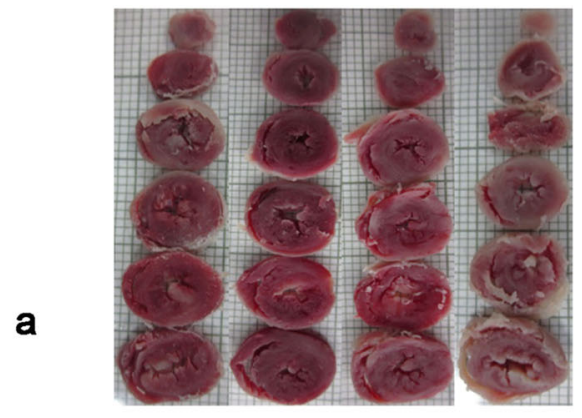

b
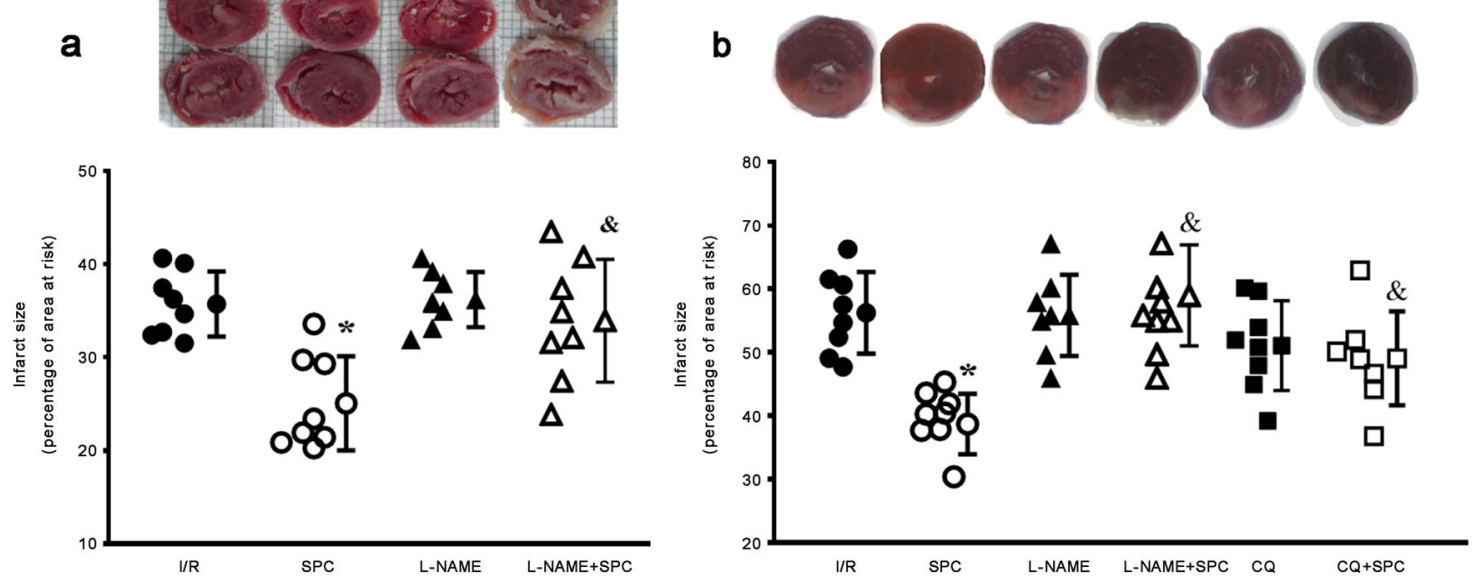

Fig. 2 SPC-induced decreases in infarct size were blocked by L-NAME and CQ in the ex vivo and in vivo models. TTC-stained images of myocardial infarct size expressed as a percentage of the area at risk (a, ex vivo and $\mathbf{b}$, in vivo). Anesthetized rats underwent 30 min of ischemia followed by $2 \mathrm{~h}$ of reperfusion (I/R) with or without sevoflurane treatment Means \pm SD. ${ }^{*} P<0.05$ vs. I/R group; ${ }^{\&} P<0.05$ vs. SPC group ( $n=8 /$ group) 

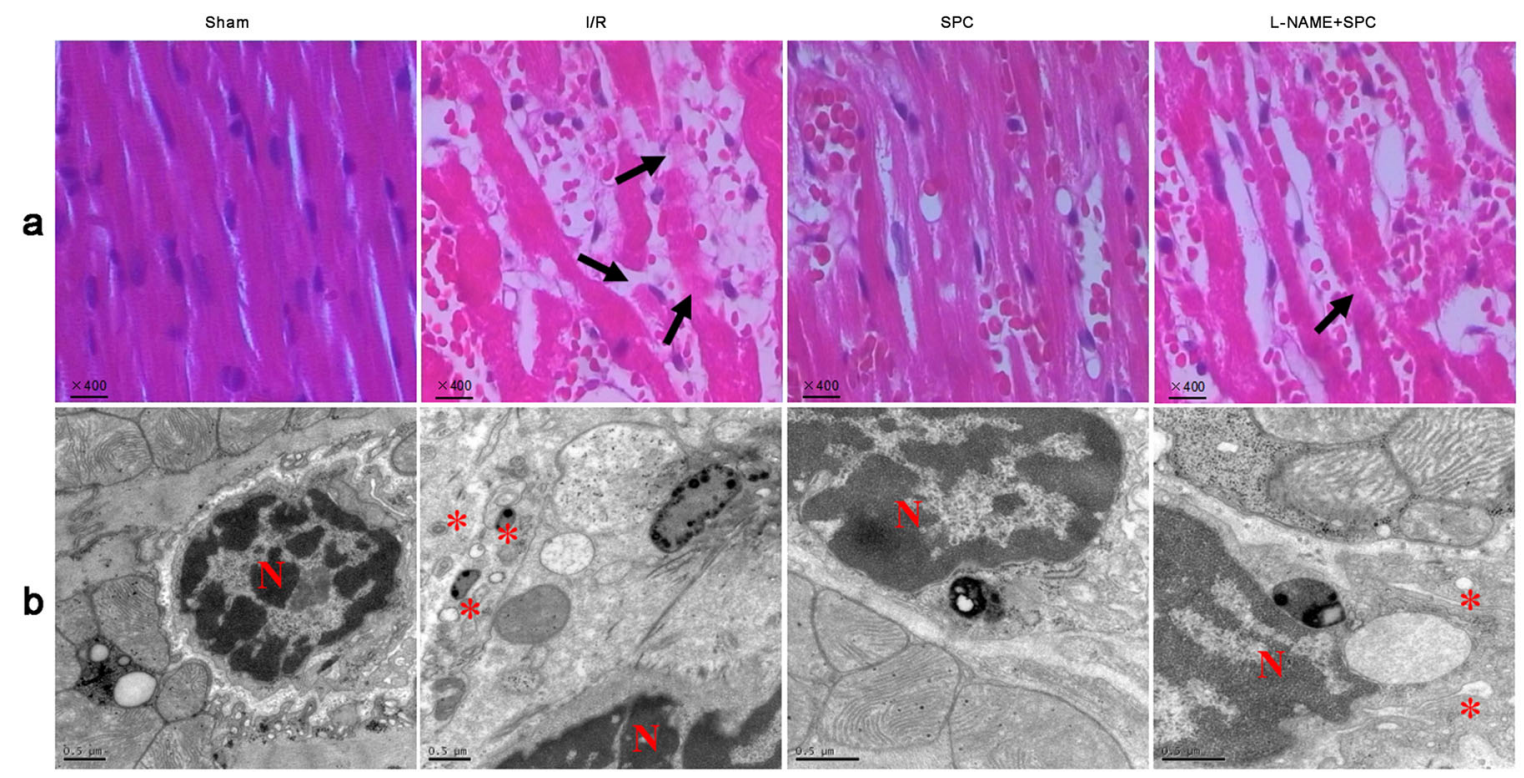

Fig. 3 Sevoflurane postconditioning (SPC) attenuated histopathologic damage and ultrastructure changes following I/R in vivo. Left ventricle (LV) tissues were retrieved at the end of reperfusion, and paraffin sections were prepared and subjected to HE staining. a HE staining images (magnification, 400x) ( $n=3$ /group). b LV myocardial ultrastructure by transmission electron microscopy. Note: The arrowhead indicates that myofilaments were absent or fractured; the nuclear chromatin edge set, and an asterisk indicates mitochondrial edema near the autophagosomes ( $n=3$ /group). $N$ nuclear. Scale bar: $0.5 \mu \mathrm{m}$

\section{Histopathology}

At the end of reperfusion, the hearts were harvested, rinsed, and fixed in $10 \%$ formaldehyde solution for $24 \mathrm{~h}$. Then, the hearts were dehydrated, embedded in paraffin wax, and cut into 4- $\mu \mathrm{m}$-thick slices, as shown in Fig. 3a, which were then stained with hematoxylin-eosin (HE) as previously described [3].

Transmission electron microscopy

At the end of reperfusion, the rat hearts were rapidly removed to detect myocardial ultrastructural alterations by transmission electron microscopy (TEM), as shown in Fig. 3b. Approximately 1-mm-thick tissues were obtained from the apex cordis and were fixed in $2.5 \%$ ice-cold glutaraldehyde in $0.1 \mathrm{M}$ of PBS and preserved at $4{ }^{\circ} \mathrm{C}$ for further processing. After $24 \mathrm{~h}$, the tissue blocks were post-fixed in $1 \%$ osmium tetroxide for $2 \mathrm{~h}$, dehydrated in graded alcohol solutions, and subsequently embedded in Epon 812. Areas of interest were selected from toluidine-blue-stained semi-thin sections, and then ultrathin sections were mounted in slot grids, covered with formalin, and double-stained in uranyl acetate and lead citrate. The sections were examined via TEM (Technai 10; Philips, the Netherlands) at magnifications ranging from $\times 3000$ to $\times 15,000$ to assess the ultrastructural features of cardiomyocytes [20].

Determination of NOS, NO, and NAD ${ }^{+}$levels

After $2 \mathrm{~h}$ of reperfusion, hearts were removed and homogenized in $0.9 \%$ ice-cold saline solution and centrifuged at $600 \times g$ for $10 \mathrm{~min}$. NOS and NO levels were measured using a diagnostic assay kit (Nanjing Jiancheng Institute of Biological Engineering, Nanjing, China). Absorbance was measured using a DU-640 spectrophotometer (Beckman Coulter Inc., Pasadena, CA, USA) at $530 \mathrm{~nm}$ and was plotted as a percentage of the control according to the manufacturer's instructions [4]. In another group, hearts were collected after $5 \mathrm{~min}$ of ischemia for nicotinamide adenine dinucleotide $\left(\mathrm{NAD}^{+}\right)$measurement [22]. $\mathrm{NAD}^{+}$was extracted and diluted in alcohol dehydrogenase, and its activity was determined using a multi-frequency phase spectrofluorometer (DU-640, Beckman Coulter Inc.) to fluorometrically measure NAD ${ }^{+}$dependent lactate dehydrogenase activity in a reaction buffer at a wavelength of $340 \mathrm{~nm}$.

\section{Immunoblotting}

Hearts were homogenized using RIPA lysis buffer (20-188, Millipore, Billerica, MA, USA) and a complete mammalian proteinase inhibitor cocktail (PI101, Roche Diagnostics $\mathrm{GmbH}$, Mannheim, Germany) and then centrifuged at 13,200×g for $20 \mathrm{~min}$ at $4{ }^{\circ} \mathrm{C}$. The protein content of the samples was determined using a bicinchoninic acid assay kit (P0010, Biyuntian Biotechnology Company, China) and was adjusted to the same concentration with RIPA. After denaturation, $20 \mu \mathrm{g}$ of each sample was electrophoresed in 8-12\% SDS-polyacrylamide gels (Bio-Rad, USA) and then transferred to polyvinylidene fluoride membranes. After blocking with $5 \%$ skim milk for $2 \mathrm{~h}$, the membranes were incubated at $4{ }^{\circ} \mathrm{C}$ overnight with the following primary antibodies: microtubule-associated protein 1 light chain 3 (LC3, Abcam, Cambridgeshire, UK), Beclin 1 (Cell Signaling Technology, Danvers, MA, USA), lysosome-associated membrane protein-2 (LAMP-2, Santa Cruz Biotechnology, California, USA), P62 (Sigma-Aldrich, St. Louis, USA), cytochrome $c$, eNOS (Santa Cruz Biotechnology, Texas, USA), nNOS (Santa Cruz Biotechnology, Texas, USA) and the housekeeping protein GAPDH (Biyuntian Biotechnology Company, China). Then, the membranes were incubated with peroxidaseconjugated secondary antibodies for $2 \mathrm{~h}$. Immunoreactive bands were visualized by enhanced chemiluminescence and images were assessed using densitometric analysis software $[15,20,21]$.

\section{Statistical analysis}

The GraphPad Prism 5.0 statistical software (GraphPad Software, Inc, San Diego, USA) was used for statistical processing. All data are expressed as the means \pm SD. One-way or two-way analysis of variance (ANOVA) was used for the statistical analysis of data obtained within the same group of rats and among the different groups of rats, respectively, followed by the Bonferroni post hoc test for multiple comparisons of group means. $P<0.05$ was considered statistically significant.

\section{RESULTS}

The roles of NOS and autophagic flux in the effects of SPC on myocardial I/R-induced infarct size

In the isolated rat model of myocardial I/R injury, SPC significantly decreased infarct size $(25.08 \pm 5.05 \%$ in the SPC 
group vs. $35.73 \pm 3.49 \%$ in the I/R group, $n=8, P<0.05$ ) (Fig. 2a). This favorable effect of SPC was blocked by the NOS inhibitor, L-NAME, as indicated by the SPC-induced decreases in infarct size $(33.94 \pm 6.58 \%$ in the L-NAME + SPC group vs. $25.08 \pm 5.05 \%$ in the SPC group, $n=8, P<0.05)$. Similar results were found in the in vivo study; SPC reduced the postischemic IS/AAR from $56.23 \pm 6.44 \%$ to $38.71 \pm 4.78 \%(P<0.05$, SPC group vs. I/R group). Administration of either L-NAME or the autophagic flux inhibitor CQ alone had no significant impact on infarct size. Both L-NAME and CQ abolished the effect of SPC-induced infarct size reduction (Fig. 2b).

The effects of SPC on histopathological changes

To evaluate whether SPC improved cardiac histopathological and ultrastructural damage, we examined the myocardium via HE staining and TEM in intact rat hearts. As shown in Fig. 3a, the myocardial structure was arranged regularly in the group, and cardiomyocytes exhibited a normal size and clear boundaries and were arranged regularly. The postischemic myocardium exhibited an aberrant and wavy arrangement, myocardial fibers were irregular and their outlines were difficult to visualize in certain areas, and transverse striation was unclear or disordered. In addition, in the I/R rat hearts, the outlines of the myocardial fibers were hardly recognizable in some areas, and cardiomyocytes exhibited edema with neutrophil infiltration and large necrotic areas. These pathologic changes were ameliorated by SPC as indicated by better preservation of the striations, myocardial fibers, and myocytes in sevoflurane-treated rat hearts. However, the SPC-induced histological outcomes were largely abolished by the NOS inhibitor L-NAME.

Ultrastructural sections of the hearts from the group showed well-arranged sarcomeres, normal myofibrils, and mitochondrial structures with intact cristae density and uniformly scattered glycogen granules. I/R injury resulted in marked ultrastructural damage, and substantially more autophagosomes were observed. Electron photomicrographs revealed mild rarefaction of myofilaments in the SPC group. The effects of SPC in preserving ultrastructural integrity were abolished by L-NAME (Fig. 3b).

The effects of SPC on cardiac function performance

Figure 4 shows time-dependent changes in cardiac function in isolated hearts. The baseline values of LVDP, LVEDP and $\pm d P / d t$ in each group were similar $(P>0.05)$. Global ischemia resulted in systolic and diastolic dysfunction. The LVDP values gradually decreased and LVEDP increased with ischemia time. LVDP and $\pm \mathrm{d} P / \mathrm{d} t$ were significantly higher in the SPC group compared to those in the I/R group $(P<0.05)$. The LVEDP values in all groups gradually decreased with reperfusion and were significantly lower in the SPC group compared to those in the $\mathrm{I} / \mathrm{R}$ group $(P<0.05)$. L-NAME administration abolished the above beneficial effects of SPC.
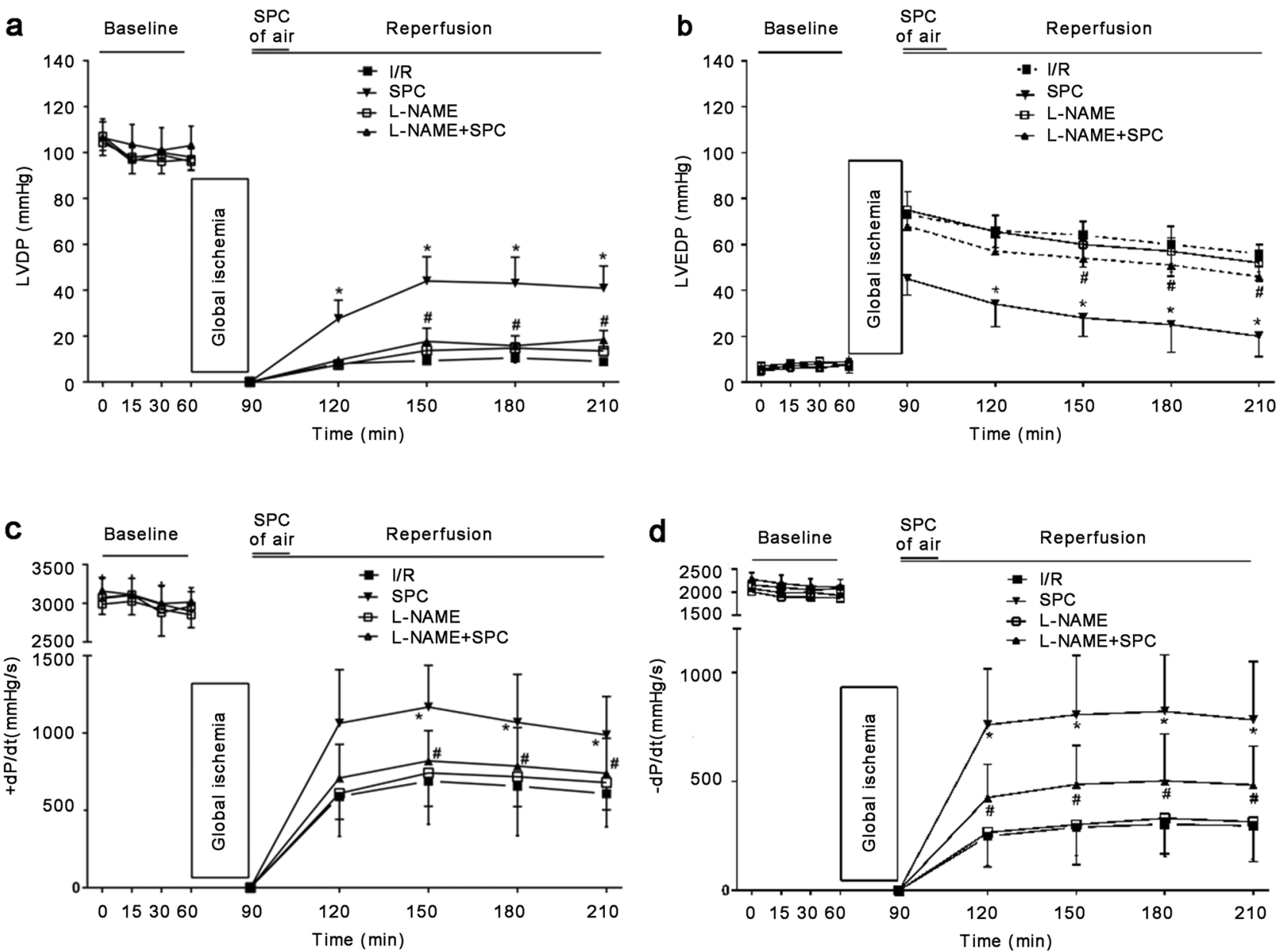

Fig. 4 SPC-induced improvements in cardiac function were blocked by the NOS inhibitor L-NAME in ex vivo studies. a Left ventricular developed pressure (LVDP). b Left ventricular end-diastolic pressure (LVEDP). $\mathbf{c}+\mathrm{d} P / \mathrm{d} t$ (maximum rate of increase in left ventricular developed pressure). $\mathbf{d}-\mathrm{d} P / \mathrm{d} t$ (maximum rate of decrease in left ventricular developed pressure). The data are presented as the means $\pm \mathrm{SD}$. ${ }^{*} P<0.05$ vs. I/R group; ${ }^{\#}<0.05$ vs. SPC group ( $n=8$ /group) 

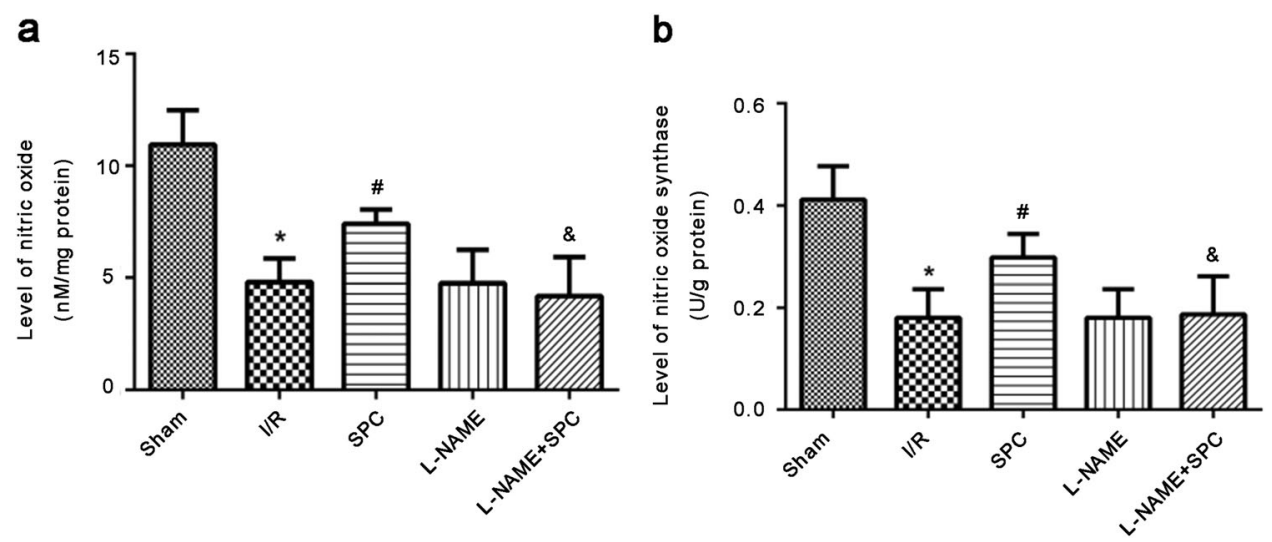

Fig. 5 Sevoflurane postconditioning (SPC) increased NO and NOS production in ex vivo experiments. SPC increased the NO and NOS contents $(\mathbf{a}, \mathbf{b})$. The SPC-induced increases in NO and NOS were inhibited by L-NAME administration. The data are presented as the means \pm SD. ${ }^{*} P<0.05$ vs. Sham group; ${ }^{\#} P<0.05$ vs. I/R group; ${ }^{\&} P<0.05$ vs. SPC group ( $n=6$ hearts/group)
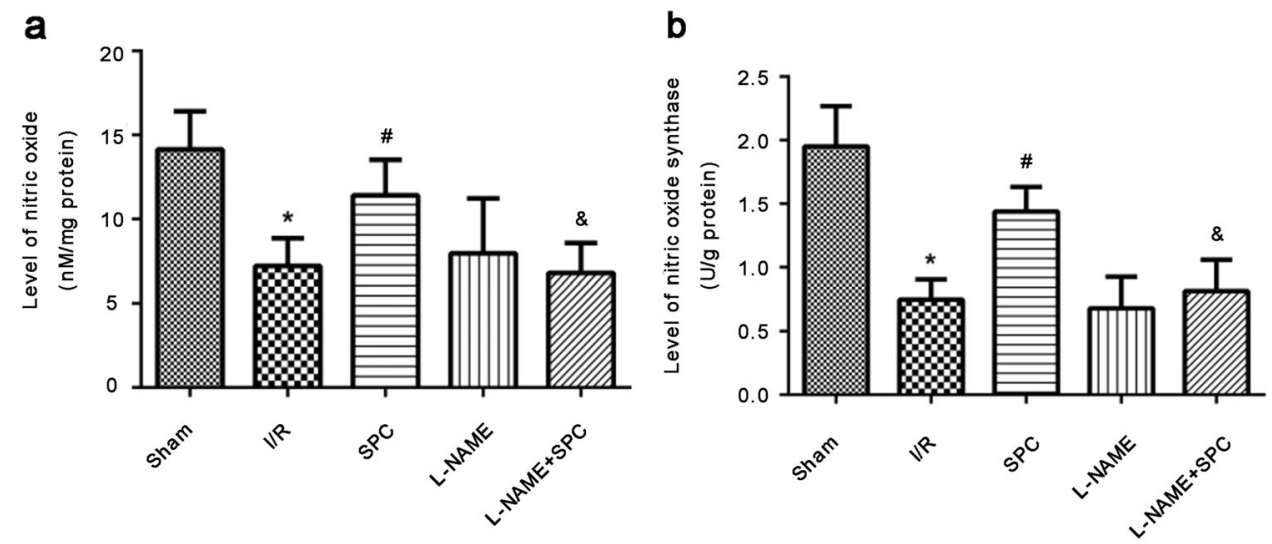

Fig. 6 Sevoflurane postconditioning (SPC) increased NO and NOS production in vivo. a SPC increased NO production. b SPC increased NOS production. SPC-induced NO and NOS production was inhibited by L-NAME treatment. The data are presented as the means \pm SD. ${ }^{*} P<0.05$ vs. Sham group; ${ }^{\#} P<0.05$ vs. I/R group; ${ }^{\&} P<0.05$ vs. SPC group ( $n=6$ hearts/group)

a
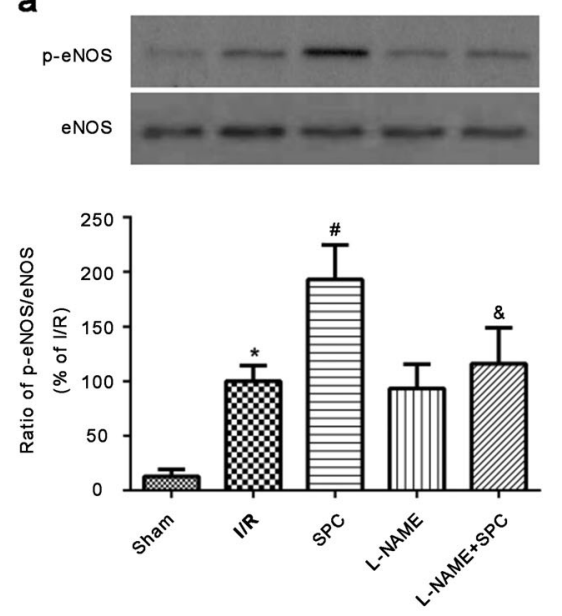

b
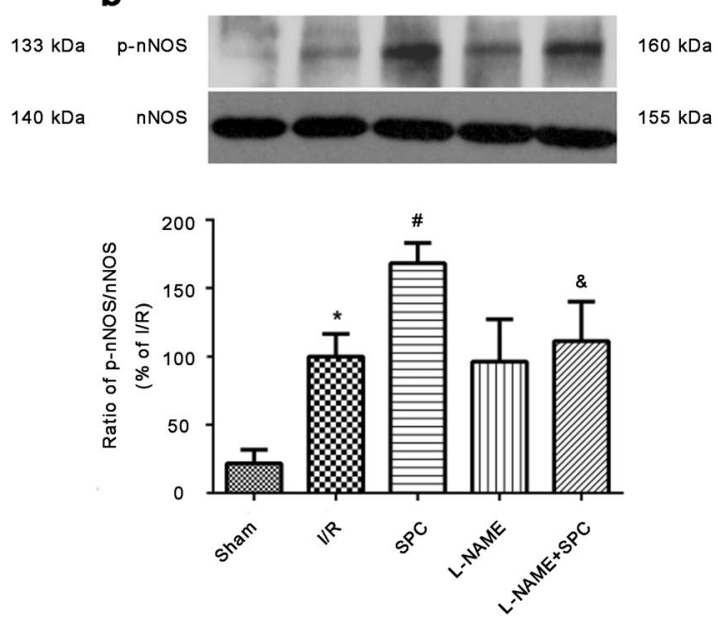

Fig. 7 SPC-induced increases in the phosphorylation of endothelial nitric oxide synthase (eNOS) and neuronal nitric oxide synthase (nNOS) were abolished by the NOS inhibitor L-NAME in ex vivo experiments. a Western blot bands of eNOS and phosphorylated eNOS (p-eNOS) and the ratio of p-eNOS/total eNOS. b Western blot bands of nNOS and phosphorylated nNOS (p-nNOS) and the ratio of $p$-nNOS/total nNOS. The data are presented as the means \pm SD. ${ }^{*} P<0.05$ vs. Sham group; ${ }^{\#} P<0.05$ vs. I/R group; ${ }^{*} P<0.05$ vs. SPC group $(n=5$ hearts/group) 
The effects of SPC on NOS activity and NO content

After $2 \mathrm{~h}$ of reperfusion, NOS activity in rat heart tissues was determined by spectrophotometry and the NO level was measured by nitrate reductase in both models. The levels of NOS and NO were both decreased in the postischemic group compared with those in the group $(P<0.05)$. NOS activity and the NO content were elevated in the SPC group compared to those in the I/R group $(P<0.05)$, but L-NAME abolished these SPC-induced NOS and NO increases. No significant differences were observed between the L-NAME group and the L-NAME + SPC group $(P>0.05)$. These results indicated that both NOS activity and the level of NO were decreased with I/R injury, but they were increased by sevoflurane treatment.
This effect of SPC was reversed by L-NAME administration (Figs. 5 and 6).

The effects of SPC on eNOS and nNOS phosphorylation and autophagic flux

The total protein expression levels of eNOS and nNOS did not change in different groups during $2 \mathrm{~h}$ of reperfusion in the ex vivo myocardial I/R model (Fig. 7). However, significant increases in phosphorylated eNOS ( $p$-eNOS) and nNOS ( $p$-nNOS) were detected in the I/R group compared to the corresponding levels in the group $(P<0.05)$. SPC further increased p-eNOS and p-nNOS levels $(P<0.05$, SPC group vs. I/R group, $n=5)$, but this effect was significantly abrogated by L-NAME administration $(P<0.05$, a
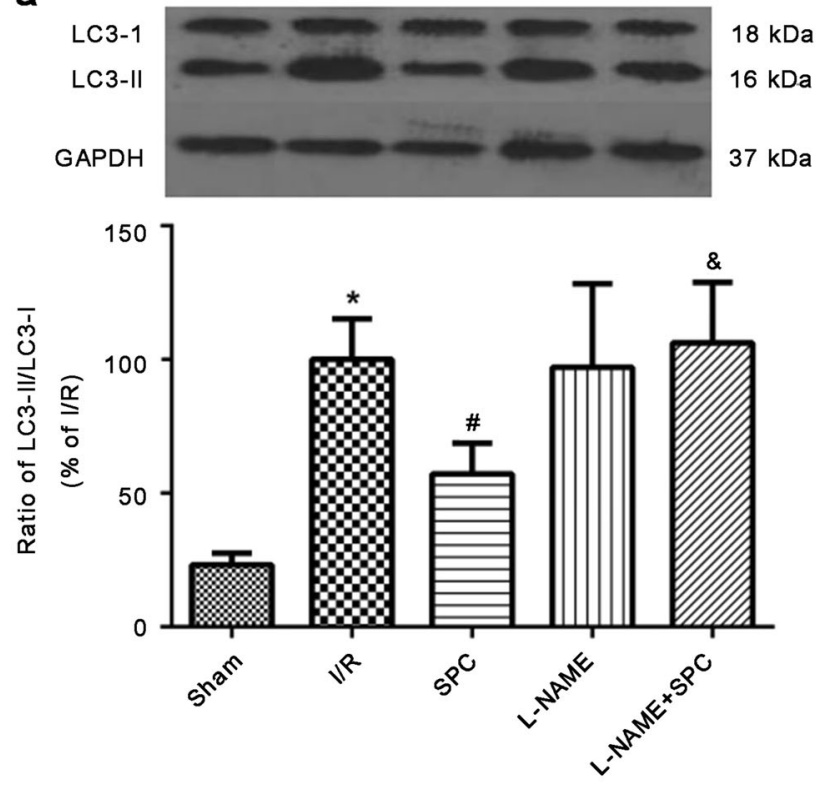

C
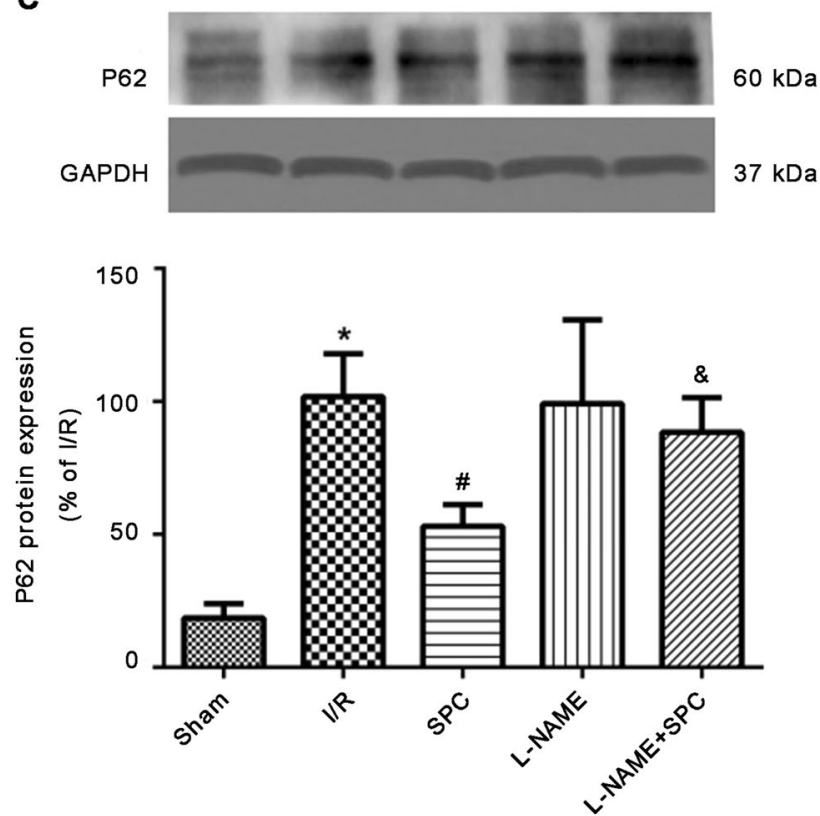

b
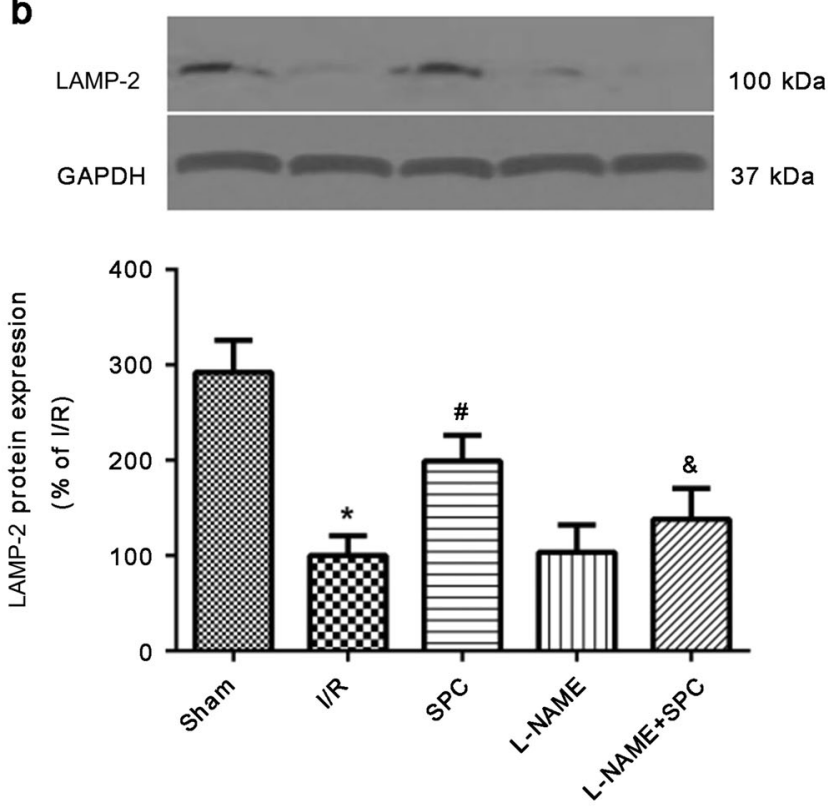

d
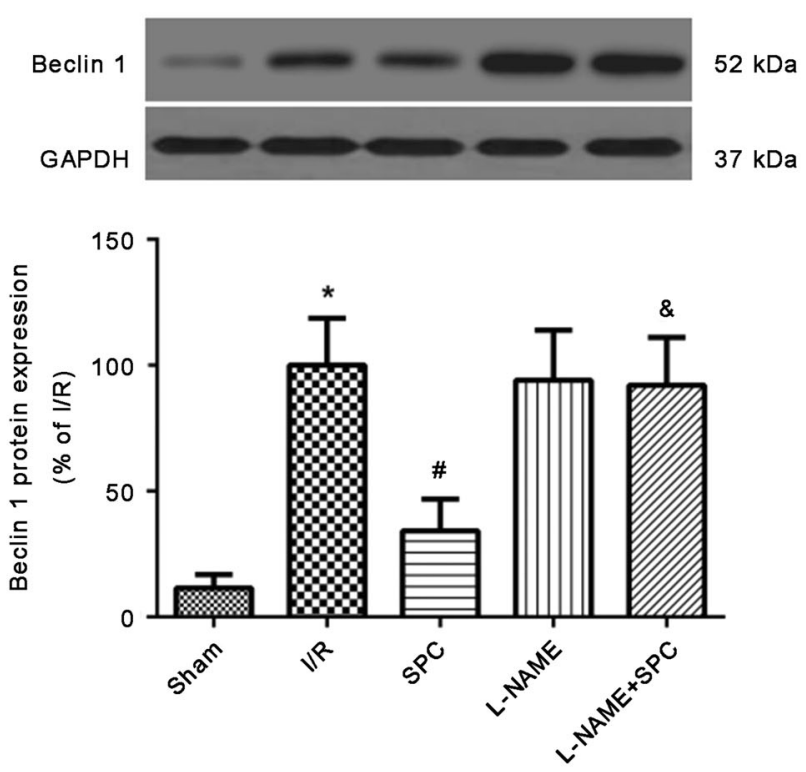

Fig. 8 SPC-enhanced autophagic flux was blocked by the NOS inhibitor L-NAME in ex vivo experiments. a Immunoblots and densitometric analysis of LC3-I and LC3-II in heart tissues. b Immunoblots and densitometric analysis of LAMP-2 in heart tissues. c Immunoblots and densitometric analysis of P62 in heart tissues. d Immunoblots and densitometric analysis of Beclin 1 in heart tissues. The data are presented as the means \pm SD. ${ }^{*} P<0.05$ vs. Sham group; ${ }^{\#} P<0.05$ vs. I/R group; ${ }^{\circledR} P<0.05$ vs. SPC group ( $n=5$ hearts/group) 
a
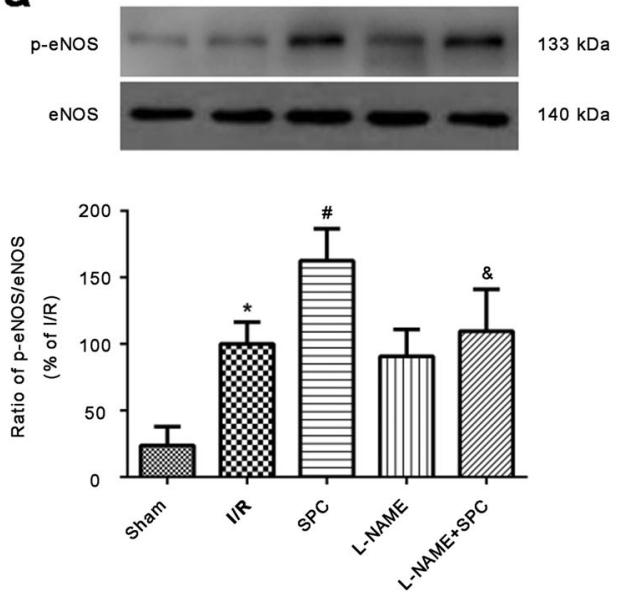

b
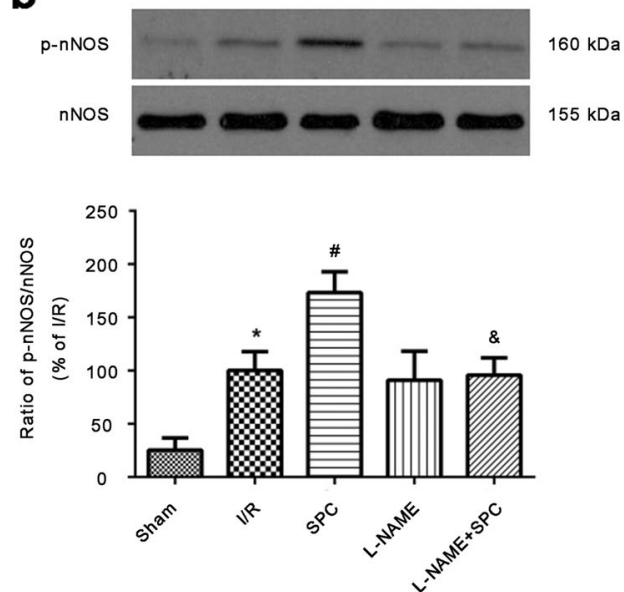

Fig. 9 SPC-induced increases in the phosphorylation of eNOS and nNOS. These effects of SPC were abolished by the NOS inhibitor L-NAME in the in vivo model. a Western blot bands of eNOS and p-eNOS and the ratio of p-eNOS/total eNOS. $\mathbf{b}$ Western blot bands of $\mathrm{nNOS}$ and $\mathrm{p}$-nNOS and the ratio of $p$-nNOS/total nNOS. The data are presented as the means \pm SD. ${ }^{*} P<0.05$ vs. Sham group; ${ }^{\#} P<0.05$ vs. I/R group; ${ }^{\&} P<0.05$ vs. SPC group ( $n=5$ hearts/group)

L-NAME + SPC group vs. SPC group). In addition, L-NAME had no significant effect on $p$-eNOS and p-nNOS expression after reperfusion injury. No significant differences in iNOS were observed among the groups (data not shown).

Myocardial I/R increased the LC3-II/I ratio, up-regulated Beclin 1 and P62 protein levels, but reduced LAMP-2 protein levels in the ischemic heart after $2 \mathrm{~h}$ of reperfusion (Fig. 8), suggesting that myocardial I/R-induced autophagosome formation and impaired autophagosome clearance. SPC treatment at the start of reperfusion blunted the I/R-induced increases in the LC3-II/I ratio and Beclin 1 and P62 expression levels and the I/R-induced decrease in LAMP-2 expression ( $P<0.05$, SPC group vs. I/R group, $n=5)$, indicating that SPC restored I/R-impaired autophagy flux. These effects of SPC were blunted by L-NAME administration.

Similar results were obtained in the ex vivo study in which the cardiac expression of $\mathrm{p}$-eNOS and p-nNOS, the LC3-II/I ratio, and Beclin 1 and P62 protein levels were significantly increased, but LAMP-2 protein levels were reduced in the I/R group. SPC further enhanced p-eNOS, p-nNOS, and LAMP-2 levels but decreased the LC3-II/I ratio and Beclin 1 and P62 protein levels. L-NAME administration abolished these SPC-induced changes (Figs. 9 and 10).

The effects of SPC on mitochondrial permeability transition pore (mPTP) opening and cytochrome $c$ release

In this study, we found that SPC significantly increased the mitochondrial NAD ${ }^{+}$level $(P<0.05$, SPC group vs. I/R group, $n=5)$ and decreased cytochrome $c$ expression as evidenced by Western blot. However, L-NAME completely abolished the SPC-mediated increase in mitochondrial $\mathrm{NAD}^{+}$and decrease in cytosolic cytochrome $c$ release (Fig. 11).

\section{DISCUSSION}

The results of this study demonstrate that NO plays a critical role in the cardioprotective effects of SPC. SPC alleviated I/R-induced cardiac dysfunction, reduced the postischemic infarct size, and resulted in higher eNOS and nNOS phosphorylation levels, NOS content, and NO production. SPC also decreased mPTP opening and cytochrome $c$ release and restored I/R-impaired autophagic flux. These beneficial effects of SPC are lost with administration of the NOS inhibitor L-NAME and the autophagic flux blocker CQ. The results provide evidence that SPC attenuates myocardial reperfusion injury by restoring l/R-impaired autophagic flux through an NO-dependent mechanism.

NO is a unique, endogenous regulatory molecule involved in various physiological processes. NO exerts some beneficial effects during myocardial reperfusion, including regulation of myocardial contractility, as well as effects on sarcolemmal and mitochondrial $\mathrm{K}_{\text {ATP }}$ channel opening, antioxidant effects, and oxygen free radical production [23, 24]. Although myocardial I/R may impair NO release, isoflurane exposure has been found to stimulate vascular endothelial cells to produce more NO in the ischemic-reperfused myocardium [16]. This study showed that NOS activity and the NO content in the ischemic myocardium were obviously lower than those in the nonischemic myocardium. After sevoflurane was administered at the onset of reperfusion, NOS activity and NO production were higher than the corresponding levels in the $I / R$ group, which is consistent with previous reports $[2,16]$. NO may be produced from three different NOS isoforms in cardiac myocytes following I/R injury, including nNOS, iNOS and eNOS, whereas it may be predominantly derived from eNOS rather than other NOS isoforms in pharmacological postconditioning-induced cardioprotection against acute I/R injury $[16,25]$. The non-specific NOS inhibitor L-NAME was used to inhibit all isoforms of NOS during myocardial I/R in our study, abolishing the observed SPCinduced improvements in cardiac function recovery, infarct size reduction, and higher eNOS and nNOS expression levels. In addition, iNOS expression did differ between the SPC group and the $I / R$ group in this study.

The mechanisms through which NO protects against myocardial I/R injury are related to mitochondria. Mitochondria are principal energy providers for cells, and mitochondrial damage results in increased mPTP opening, reactive oxygen species (ROS) production and cytochrome $c$ release, ultimately resulting in cell death. Well-balanced mitochondrial function plays a key role in controlling cell survival $[26,27]$. NO can bind to cytochrome $c$ oxidase with an affinity similar to that for guanylate cyclase and inhibit complex I and Fe-S centers by limiting mitochondrial respiration to prevent abrupt mPTP opening [28]. mPTP opening leads to mitochondrial membrane potential collapse, respiratory chain uncoupling, $\mathrm{Ca}^{2+}$ and cytochrome $c$ efflux, and matrix swelling [29]. Previous studies have shown that delayed mPTP opening reduced the $\mathrm{NAD}^{+}$content of cytoplasm [30]. The level of $\mathrm{NAD}^{+}$was considered a good marker for MPTP opening in myocardial $\mathrm{I} / \mathrm{R}$ heart tissues, and it was usually drastically 
a
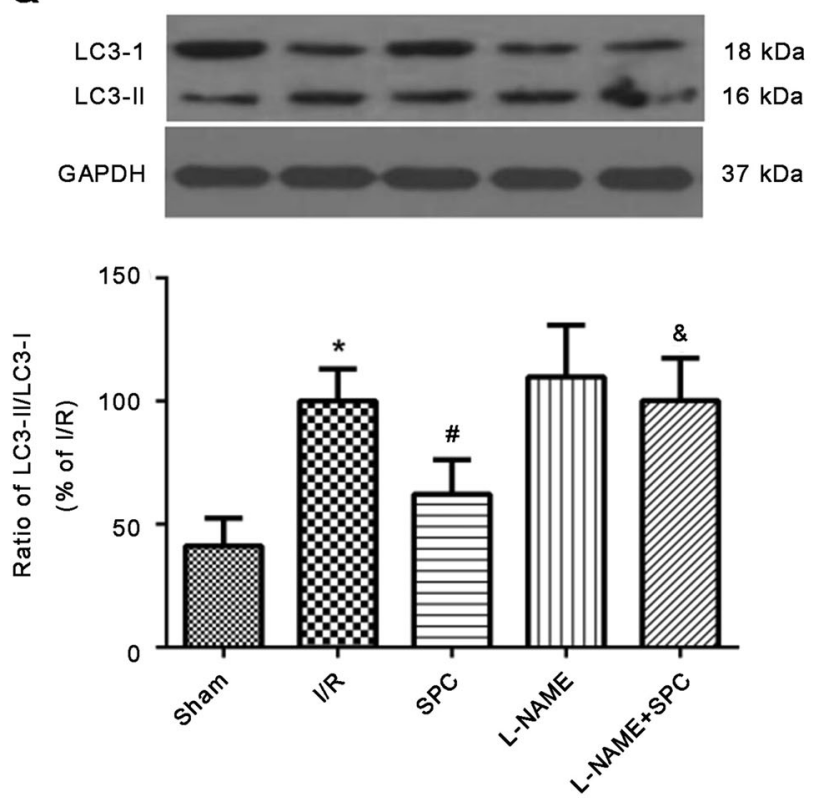

C
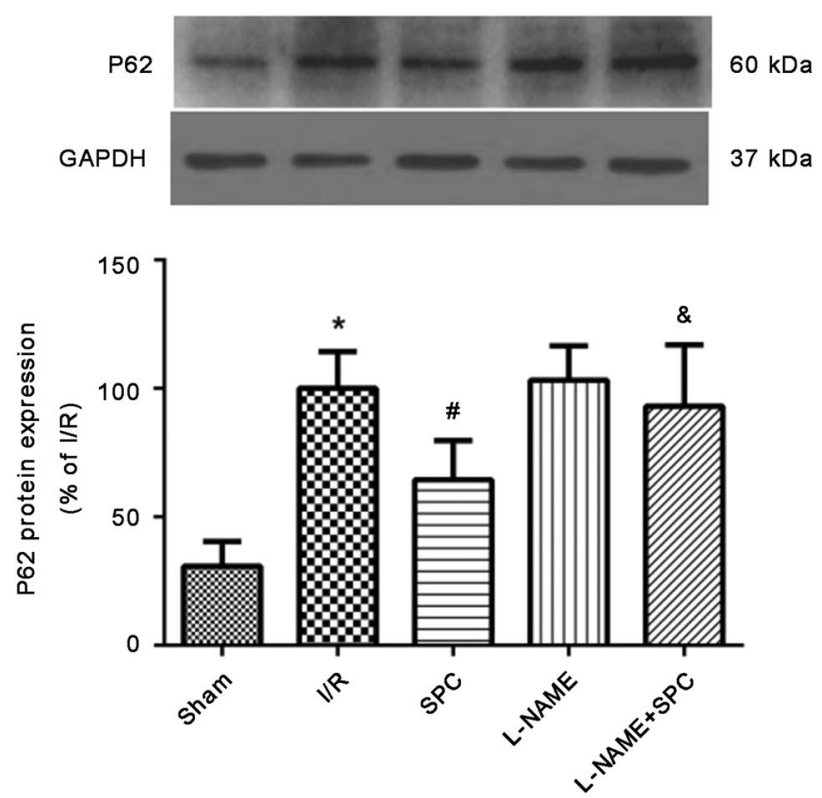

b
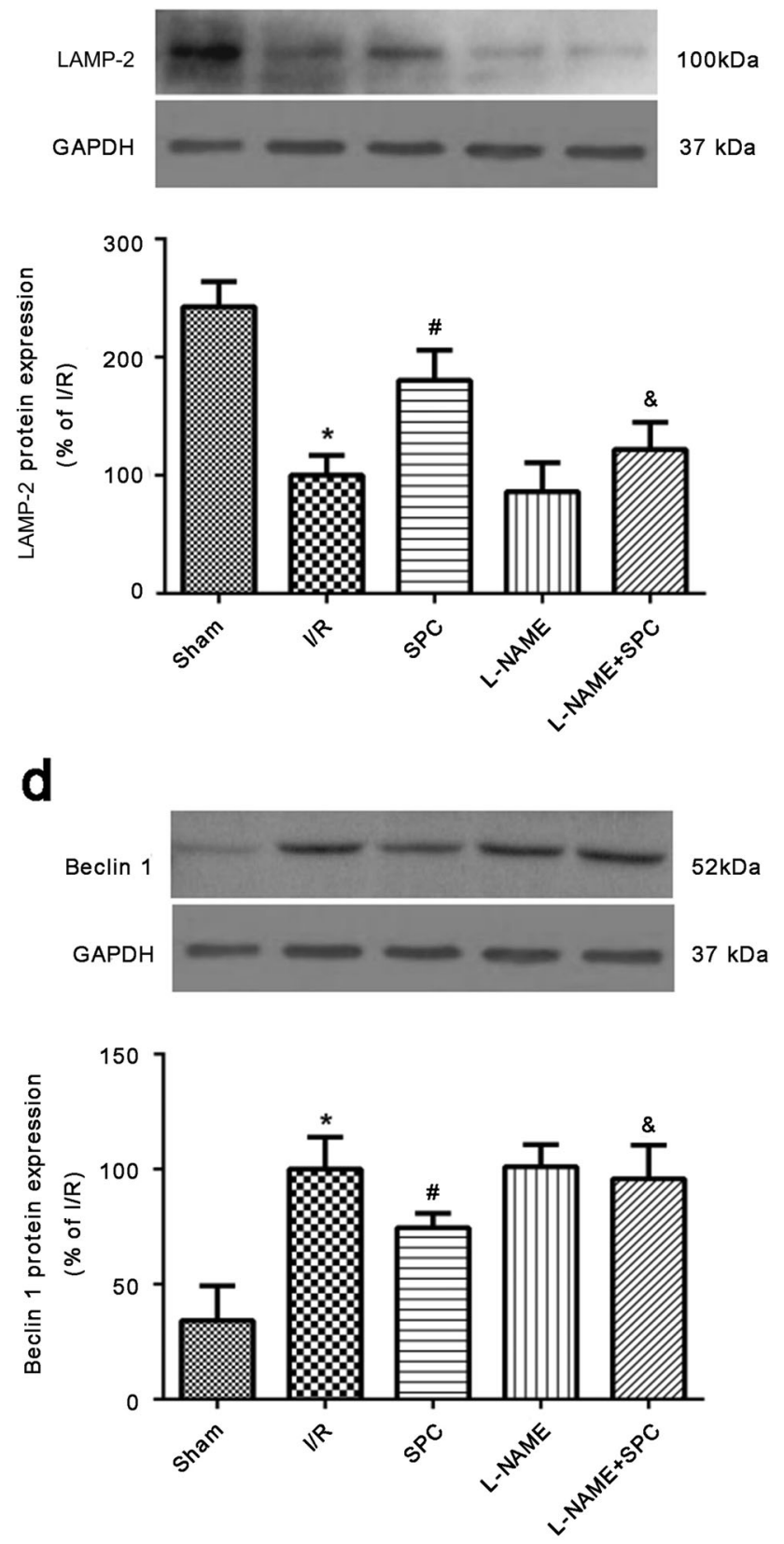

Fig. 10 SPC-enhanced autophagic flux was blocked by the NOS inhibitor L-NAME in the in vivo model. a Immunoblots and densitometric analysis of LC3-I and LC3-II in heart tissues. b Immunoblots and densitometric analysis of LAMP-2 in heart tissues. c Immunoblots and densitometric analysis of P62 in heart tissues. d Immunoblots and densitometric analysis of Beclin 1 in heart tissues. The data are presented as the means \pm SD. ${ }^{*} P<0.05$ vs. Sham group; ${ }^{\sharp} P<0.05$ vs. I/R group; ${ }^{\&} P<0.05$ vs. SPC group ( $n=5$ hearts/group)

decreased in the ischemic myocardium, which subsequently resulted in cytochrome $c$ release [31]. Our previous study showed that $\mathrm{NADH}$ initially increased and peaked at $5 \mathrm{~min}$ of ischemia, followed by a gradual decline below baseline levels later during ischemia [22]. In this study, SPC increased the cytoplasmic content of $\mathrm{NAD}^{+}$and decreased cytochrome $c$ release. The beneficial effects of SPC on hearts subjected to $\mathrm{I} / \mathrm{R}$ are likely related to mitochondrial protection. Interestingly, the protective effects of SPC on mitochondria were abolished by L-NAME, suggesting that mitochondria are a target of SPC-derived NO. Future studies should examine the impact of SPC on the production of highenergy phosphates and superoxide and mPTP opening in the postischemic myocardium.
Most studies on the mechanisms responsible for I/R-induced cardiac abnormalities have focused on necrosis and apoptosis. Recently, dysregulated autophagy was shown to have unique roles in reperfusion injury and post-infarction cardiac remodeling [5]. Autophagy is a dynamic intracellular lysosomal degradative process in which cargo is engulfed by double membrane-bound autophagosomes, and then the autophagosomes fuse with lysosomes for subsequent degradation [32]. Complete autophagic flux is the determining factor in maintaining intracellular homeostasis and cell survival. Ma et al. found that myocardial autophagic flux was impaired following l/R-induced myocardial infarction during the reperfusion phase. The LC3-II/I ratio is a wellestablished marker of autophagosome formation. LAMP-2, a 

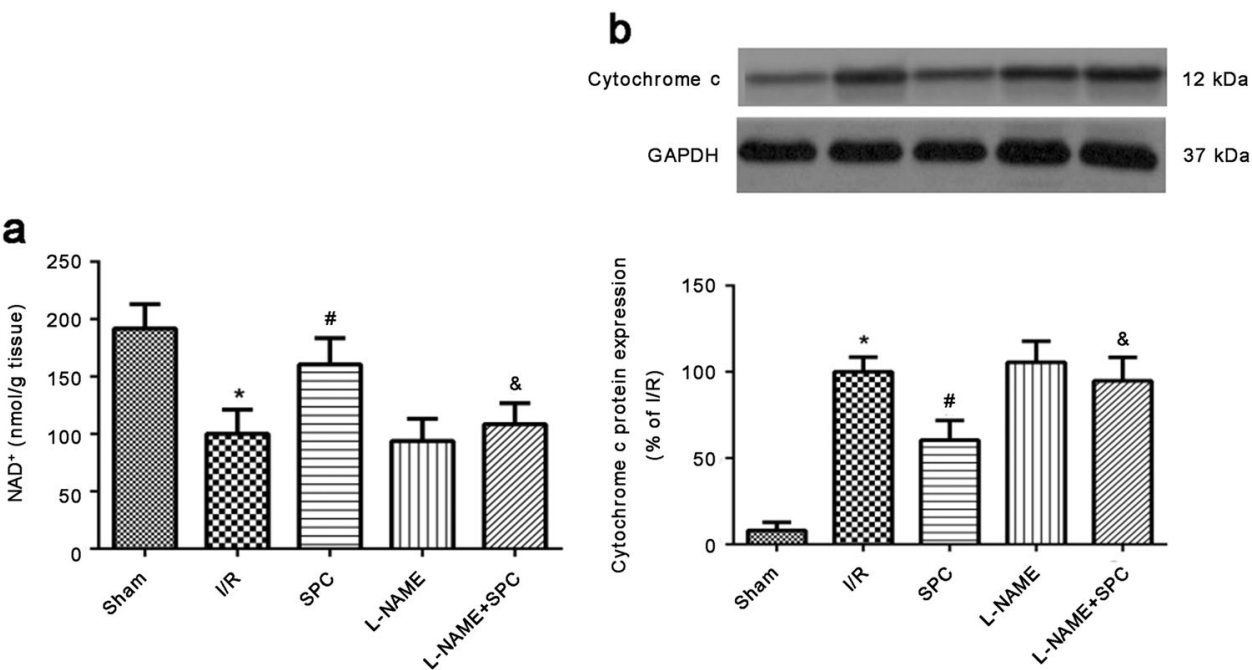

Fig. 11 SPC reduced nicotinamide adenine dinucleotide $\left(\mathrm{NAD}^{+}\right)$content loss (a) and cytochrome $c$ release (b) due to l/R in the in vivo model. SPC-induced mitochondrial protection was inhibited by L-NAME treatment. The data are presented as the means \pm SD. ${ }^{*} P<0.05$ vs. Sham group; ${ }^{\#} P<0.05$ vs. I/R group; ${ }^{\circledR} P<0.05$ vs. SPC group ( $n=5$ hearts /group)

critical determinant of autophagosome-lysosome fusion, is essential for autophagosome clearance and autophagic flux [33]. Beclin 1 is well known to mediate autophagy up-regulation during reperfusion. A previous study showed that myocardial $\mathrm{I} / \mathrm{R}$ impairs autophagic flux by up-regulating Beclin 1 and downregulating LAMP-2 [11]. Moreover, P62, an adapter protein that links aggregated proteins sequestered in autophagosomes and degraded in autolysosomes, has been used as an index of autophagic flux [32]. Blocking the fusion of autophagosomes and lysosomes results in autophagosome accumulation, which exacerbates myocardial reperfusion injury [11]. A previous study demonstrated that SPC improved postischemic mitochondrial function $[9,34]$. Recently, several studies have reported that the autophagosome accumulation induced by $\mathrm{CQ}$ increases mitochondrial membrane potential loss and deleterious autophagic processes [35, 36]. In contrast, autophagy can reduce ROS production by removing damaged mitochondria and thus decrease MPTP opening, ROS production and cytochrome $c$ release [7]. In this study, the numbers of autophagosomes in the ischemic myocardium were obviously increased, as well as LC3-II, Beclin 1, and P62 levels, suggesting that myocardial I/R-induced autophagosome formation. A previous study reported that reperfusion injury impaired autophagic flux and that excessive autophagosome accumulation induced cell death [11]. In this study, after SPC treatment, the levels of LC3-II, Beclin 1, and P62 were decreased, cardiac function performance was improved, and myocardial infarct size was reduced. Lysosomal protease LAMP-2 expression was also increased after SPC treatment. Both the autophagic flux inhibitor CQ and the NOS inhibitor L-NAME abolished SPC-induced cardioprotection. NO can inhibit autophagy by suppressing JNK in meniscal cells [37], and Netrin-1 has been shown to improve post-injury cardiac function through colorectal cancer (DCC)/NO-dependent preservation of mitochondrial integrity and thus attenuate autophagy [38]. Park et al. [17] reported that nitric oxide mediated autophagy and reduced human dental pulp cell death by activating the AMPK pathway. These studies indicated that NO may induce autophagy production. However, the relationship between $\mathrm{NO}$ and autophagy in the protective effect of SPC on myocardial reperfusion injury remains unknown. Our study demonstrated that the protective effect of SPC against reperfusion injury was mediated by restoration of intact autophagic flux, which was characterized by a decline in autophagosome accumulation and attenuated autophagic markers. Our data also showed that NO inhibition caused and LC3 up-regulation and increased P62, indicating that the eNOS/NO pathway is involved in the SPC-induced changes in autophagic flux.

Some limitations exist in this study. First, an interaction between mitochondrial dysfunction and autophagy was not observed. Although mitochondrial dysfunction and autophagy reportedly determine cell survival, the crosstalk between autophagy and mitochondria underlying myocardial $\mathrm{I} / \mathrm{R}$ injury is unknown and requires further investigation [9]. Second, autophagic indicators after administration of the autophagic flux blocker CQ were not examined in this study. In the intact rat heart, CQ blocked SPCmediated attenuation of myocardial I/R-induced infarct size, and Zhang et al. [12] demonstrated that the LC3-II/I ratio and P62 level were increased after CQ treatment, which is consistent with our results. Third, we used L-NAME, which is a non-specific NOS inhibitor, and its inhibitory effects on NO production may not affect eNOS alone. However, for NO mainly derived from eNOS, L-NAME can reportedly inhibit NO release and play an important role in anesthetic-induced cardioprotection against acute reperfusion injury [16]. Specific eNOS inhibitors, such as L-NIO, should be used to identify the mechanism of eNOS involved in SPC.

In summary, this study clearly illustrates that SPC effectively protects the heart against myocardial reperfusion injury by enhancing NO production. Moreover, we provided evidence that SPC increases NO release and consequently restores I/R-impaired autophagic flux, demonstrating a potential therapeutic approach in the treatment of myocardial reperfusion injury.

\section{ACKNOWLEDGEMENTS}

This work was supported by grants SS201613 (to Dr. Wang) and SS201756 (to Dr. An) from the Suzhou Science and Technology Development Plan; BK20141187 (to Dr. Wang) from the Jiangsu Natural Science Foundation; SYS201473 (to Dr. Qiao) from the Suzhou Science and Technology Development Plan; NSFC 81703501 (to Dr. Qiao) from the National Natural Science Foundation of China; and QNRC2016219 (to Dr. Qiao) from Jiangsu Key Talent Youth Awards in Medicine.

\section{AUTHOR CONTRIBUTIONS}

S-gQ, YS, and BS conceived and designed the experiments; H-IZ, J-zA, and CW conducted the experiments; S-gQ, YS, BS, AW, JQ, and LH were involved in the interpretation of the studies, analysis of the data and review of the manuscript; S-gQ, $\mathrm{H}-\mathrm{IZ}$, and $\mathrm{CW}$ wrote the paper. 


\section{ADDITIONAL INFORMATION}

Competing interests: The authors declare no competing interests.

Publisher's note: Springer Nature remains neutral with regard to jurisdictional claims in published maps and institutional affiliations.

\section{REFERENCES}

1. Zhao Z, Corvera J, Halkos M, Kerendi F, Wang N, Guyton R, et al. Inhibition of myocardial injury by ischemic postconditioning during reperfusion: comparison with ischemic preconditioning. Am J Physiol Heart Circ Physiol. 2003;285:H579-88.

2. Cao J, Xie H, Sun Y, Zhu J, Ying M, Qiao S, et al. Sevoflurane post-conditioning reduces rat myocardial ischemia reperfusion injury through an increase in NOS and a decrease in phopshorylated NHE1 levels. Int J Mol Med. 2015;36:1529-37.

3. Huang Z, Zhong X, Irwin MG, Ji S, Wong GT, Liu Y, et al. Synergy of isoflurane preconditioning and propofol postconditioning reduces myocardial reperfusion injury in patients. Clin Sci. 2011;121:57-69.

4. Wei C, Gao J, Li M, Li H, Wang Y, Li H, et al. Dopamine D2 receptors contribute to cardioprotection of ischemic post-conditioning via activating autophagy in isolated rat hearts. Int J Cardiol. 2015;203:837-9.

5. Li H, Zhang X, Tan J, Sun L, Xu LH, Jiang YG, et al. Propofol postconditioning protects $\mathrm{H} 9 \mathrm{c} 2$ cells from hypoxia/reoxygenation injury by inducing autophagy via the SAPK/JNK pathway. Mol Med Rep. 2018;17:4573-80.

6. Guo L, Xu JM, Mo XY. Ischemic postconditioning regulates cardiomyocyte autophagic activity following ischemia/reperfusion injury. Mol Med Rep. 2015;12:1169-76

7. Hao M, Zhu S, Hu L, Zhu H, Wu X, Li Q. Myocardial ischemic postconditioning promotes autophagy against ischemia reperfusion injury via the activation of the nNOS/AMPK/mTOR pathway. Int J Mol Sci. 2017;18:614.

8. Xie M, Kong Y, Tan W, May H, Battiprolu PK, Pedrozo Z, et al. Histone deacetylase inhibition blunts ischemia/reperfusion injury by inducing cardiomyocyte autophagy. Circulation. 2014;129:1139-51.

9. Yu P, Zhang J, Yu S, Luo Z, Hua F, Yuan L, et al. Protective effect of sevoflurane postconditioning against cardiac ischemia/reperfusion injury via ameliorating mitochondrial impairment, oxidative stress and rescuing autophagic clearance. PLoS ONE. 2015;10:e0134666.

10. Hariharan $N$, Zhai $P$, Sadoshima J. Oxidative stress stimulates autophagic flux during ischemia/reperfusion. Antioxid Redox Signal. 2011;14:2179-90.

11. Ma X, Liu H, Foyil SR, Godar RJ, Weinheimer CJ, Hill JA, et al. Impaired autophagosome clearance contributes to cardiomyocyte death in ischemia/reperfusion injury. Circulation. 2012;125:3170-81.

12. Zhang YL, Yao YT, Fang NX, Zhou CH, Gong JS, Li LH. Restoration of autophagic flux in myocardial tissues is required for cardioprotection of sevoflurane postconditioning in rats. Acta Pharmacol Sin. 2014;35:758-69.

13. Fan L, Chen D, Wang J, Wu Y, Li D, Yu X. Sevoflurane ameliorates myocardial cell injury by inducing autophagy via the deacetylation of LC3 by SIRT1. Anal Cell Pathol (Amst). 2017:2017:6281285.

14. Hu L, Wang J, Zhu H, Wu X, Zhou L, Song Y, et al. Ischemic postconditioning protects the heart against ischemia-reperfusion injury via neuronal nitric oxide synthase in the sarcoplasmic reticulum and mitochondria. Cell Death Dis. 2016;7:e2222.

15. Qiao S, Olson JM, Paterson M, Yan Y, Zaja I, Liu Y, et al. MicroRNA-21 mediates isoflurane-induced cardioprotection against ischemia-reperfusion injury via Akt/ nitric oxide synthase/mitochondrial permeability transition pore pathway. Anesthesiology. 2015;123:786-98.

16. Ge ZD, Pravdic D, Bienengraeber M, Pratt PF, Auchampach JA, Gross GJ, et al. Isoflurane postconditioning protects against reperfusion injury by preventing mitochondrial permeability transition by an endothelial nitric oxide synthasedependent mechanism. Anesthesiology. 2010;112:73-85.

17. Park SY, Park MY, Park HG, Lee KJ, Kook, Kim WJ, et al. Nitric oxide-induced autophagy and the activation of AMPK pathway protect against apoptosis in human dental pulp cells. Int Endod J. 2017;50:260-70.

18. Jin L, Gao H, Wang J, Yang S, Wang J, Liu J, et al. Role and regulation of autophagy and apoptosis by nitric oxide in hepatic stellate cells during acute liver failure. Liver Int. 2017;37:1651-9.

19. Xie H, Zhang J, Zhu J, Liu LX, Rebecchi M, Hu SM, et al. Sevoflurane postconditioning protects isolated rat hearts against ischemia-reperfusion injury via activation of the ERK1/2 pathway. Acta Pharmacol Sin. 2014;35:1504-13.
20. Qiao S, Xie H, Wang C, Wu X, Liu H, Liu C. Delayed anesthetic preconditioning protects against myocardial infarction via activation of nuclear factor- $\mathrm{KB}$ and upregulation of autophagy. J Anesth. 2013;27:251-60.

21. Qiao S, Mao X, Wang Y, Lei S, Liu Y, Wang T, et al. Remifentanil preconditioning reduces postischemic myocardial infarction and improves left ventricular performance via activation of the janus activated Kinase-2/Signal transducers and activators of Transcription- 3 signal pathway and subsequent inhibition of glycogen synthase kinase-3 $\beta$ in rats. Crit Care Med. 2016;44:e131-45.

22. An J, Camara AK, Riess ML, Rhodes SS, Varadarajan SG, Stowe DF. Improved mitochondrial bioenergetics by anesthetic preconditioning during and after $2 \mathrm{~h}$ of 27 degrees $C$ ischemia in isolated hearts. J Cardiovasc Pharmacol. 2005;46:280-7.

23. Pang L, Cai Y, Tang EH, Yan D, Kosuru R, Li H, et al. Cox-2 inhibition protects against hypoxia/reoxygenation-induced cardiomyocyte apoptosis via aktdependent enhancement of iNOS expression. Oxid Med Cell Longev. 2016;2016:3453059.

24. Li XD, Yang YJ, Geng YJ, Zhao JL, Zhang HT, Cheng YT, et al. Phosphorylation of endothelial NOS contributes to simvastatin protection against myocardial no-reflow and infarction in reperfused swine hearts: partially via the PKA signaling pathway. Acta Pharmacol Sin. 2012;33:879-87.

25. Fan W, Liu Q, Zhu X, Wu Z, Li D, Huang F, et al. Regulatory effects of anesthetics on nitric oxide. Life Sci. 2016;151:76-85.

26. Venditti P, De Rosa R, Cigliano L, Agnisola C, Di Meo S. Role of nitric oxide in the functional response to ischemia-reperfusion of heart mitochondria from hyperthyroid rats. Cell Mol Life Sci. 2004;61:2244-52.

27. Lim S, Smith KR, Lim ST, Tian R, Lu J, Tan M. Regulation of mitochondrial functions by protein phosphorylation and dephosphorylation. Cell Biosci. 2016;6:25.

28. Britt RD, Sauer K, Klein MP, Knaff DB, Kriauciunas A, Yu CA, et al. Electron spin echo envelope modulation spectroscopy supports the suggested coordination of two histidine ligands to the Rieske Fe-S centers of the cytochrome b6f complex of spinach and the cytochrome bc1 complexes of Rhodospirillum rubrum, Rhodobacter sphaeroides R-26, and bovine heart mitochondria. Biochemistry. 1991;30:1892-901.

29. Kushnareva YE, Polster BM, Sokolove PM, Kinnally KW, Fiskum G. Mitochondrial precursor signal peptide induces a unique permeability transition and release of cytochrome $c$ from liver and brain mitochondria. Arch Biochem Biophys. 2001;386:251-60.

30. Ertracht $\mathrm{O}$, Malka A, Atar S, Binah O. The mitochondria as a target for cardioprotection in acute myocardial ischemia. Pharmacol Ther. 2014;142:33-40.

31. Di Lisa F, Menabò R, Canton M, Barile M, Bernardi P. Opening of the mitochondrial permeability transition pore causes depletion of mitochondrial and cytosolic $\mathrm{NAD}+$ and is a causative event in the death of myocytes in postischemic reperfusion of the heart. J Biol Chem. 2001;276:2571-5.

32. Matsui $Y$, Takagi $H, Q u$ X, Abdellatif $M$, Sakoda $H$, Asano $T$, et al. Distinct roles of autophagy in the heart during ischemia and reperfusion: roles of AMPactivated protein kinase and Beclin 1 in mediating autophagy. Circ Res. 2007;100:914-22.

33. Xie $H, X u$ Q, Jia J, Ao $G$, Sun $Y, H u L$, et al. Hydrogen sulfide protects against myocardial ischemia and reperfusion injury by activating AMP-activated protein kinase to restore autophagic flux. Biochem Biophys Res Commun. 2015;458:632-8.

34. Yang L, Xie P, Wu J, Yu J, Yu T, Wang H, et al. Sevoflurane postconditioning improves myocardial mitochondrial respiratory function and reduces myocardial ischemia-reperfusion injury by up-regulating HIF-1. Am J Transl Res. 2016;8: 4415-24.

35. Vessoni AT, Quinet A, de Andrade-Lima LC, Martins DJ, Garcia CC, Rocha CR, et al. Chloroquine-induced glioma cells death is associated with mitochondrial membrane potential loss, but not oxidative stress. Free Radic Biol Med. 2016;90: 91-100.

36. Baek SH, Noh AR, Kim KA, Akram M, Shin YJ, Kim ES, et al. Modulation of mitochondrial function and autophagy mediates carnosine neuroprotection against ischemic brain damage. Stroke. 2014;45:2438-43.

37. Shen C, Yan J, Erkocak OF, Zheng XF, Chen XD. Nitric oxide inhibits autophagy via suppression of JNK in meniscal cells. Rheumatol (Oxf). 2014;53:1022-33.

38. Bouhidel JO, Wang P, Siu KL, Li H, Youn JY, Cai H. Netrin-1 improves postinjury cardiac function in vivo via DCC/NO-dependent preservation of mitochondrial integrity, while attenuating autophagy. Biochim Biophys Acta. 2015;1852:277-89. 